\title{
Increased metabolic risk in adolescent offspring of mothers with type 1 diabetes: the EPICOM study
}

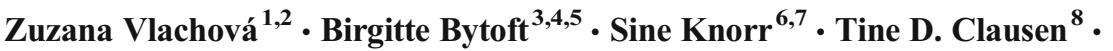 \\ Rikke Beck Jensen ${ }^{9}$ Elisabeth R. Mathiesen ${ }^{3,5,10}$ • Kurt Højlund ${ }^{1,11}$ • Per Ovesen ${ }^{12}$ • \\ Henning Beck-Nielsen ${ }^{1}$. Claus H. Gravholt ${ }^{6,7}$ • Peter Damm ${ }^{3,4,5}$ • Dorte M. Jensen ${ }^{1}$
}

Received: 11 February 2015 / Accepted: 24 March 2015 /Published online: 30 April 2015

(C) Springer-Verlag Berlin Heidelberg 2015

\begin{abstract}
Aims/hypothesis We aimed to investigate metabolic risk factors, insulin sensitivity and insulin secretion in adolescent offspring of mothers with type 1 diabetes compared with offspring of non-diabetic mothers.

Methods During 1993-1999, pregnancies of women with type 1 diabetes in Denmark were prospectively reported to a central registry in the Danish Diabetes Association. Data included information on maternal demography, diabetes status and pregnancy outcome. We invited 746 eligible children from this cohort (index offspring) to a follow-up examination. Control offspring were identified through The Danish Central Office of Civil Registration and matched with respect to date of birth, sex and postal code. Anthropometric measurements and blood sampling for metabolic characterisation, including an oral glucose tolerance test, were performed.
\end{abstract}

Electronic supplementary material The online version of this article (doi:10.1007/s00125-015-3589-5) contains peer-reviewed but unedited supplementary material, which is available to authorised users.

Zuzana Vlachová

Zuzana.Vlachova@rsyd.dk

1 Department of Endocrinology, Odense University Hospital, Kløvervænget 6, 5000 Odense C, Denmark

2 The Institute of Clinical Medicine, Faculty of Health and Medical Sciences, University of Southern Denmark, Odense, Denmark

3 Centre for Pregnant Women with Diabetes, Rigshospitalet, Copenhagen, Denmark

4 Department of Obstetrics, Rigshospital, Copenhagen, Denmark

5 The Institute of Clinical Medicine, Faculty of Health and Medical Sciences, University of Copenhagen, Copenhagen, Denmark

6 Department of Endocrinology and Internal Medicine, Aarhus University Hospital, Aarhus, Denmark
Results We examined 278 index offspring (mean age 16.7 years; range 13.0-19.8 years) and 303 control offspring (mean age 16.8 years; range 13.5-20.4 years). Index offspring had higher BMI SD score $(0.44: 95 \%$ CI $0.21,0.66)$ compared with controls, after adjustments for pubertal development and maternal pre-pregnancy BMI. Furthermore, index offspring had a higher prevalence of components included in metabolic syndrome and prediabetes (impaired fasting glucose and/or impaired glucose tolerance), with reduced insulin sensitivity and relative insulin secretion deficiency, compared with controls. Maternal $\mathrm{HbA}_{1 \mathrm{c}}$ levels in pregnancy were not directly associated with offspring metabolic outcomes.

Conclusions/interpretation Adolescent offspring of mothers with type 1 diabetes had a less favourable metabolic profile and higher frequency of prediabetes than the background population. Significant associations between these outcomes and

7 Department of Molecular Medicine, Aarhus University Hospital, Aarhus, Denmark

8 Department of Gynecology and Obstetrics, Hilleroed Hospital, Hilleroed, Denmark

9 Department of Growth and Reproduction, Rigshospitalet, University of Copenhagen, Copenhagen, Denmark

10 Department of Endocrinology, Rigshospitalet, Copenhagen, Denmark

11 Section of Molecular Diabetes \& Metabolism, Institute of Molecular Medicine, University of Southern Denmark, Odense, Denmark

12 Department of Gynecology and Obstetrics, Aarhus University Hospital, Aarhus, Denmark 
maternal $\mathrm{HbA}_{1 \mathrm{c}}$ levels in pregnancy could not be demonstrated.

Trial registration: ClinicalTrials.gov NCT01559181

Keywords Adolescent offspring - Beta cell function . Disposition Index · Fetal programming · Insulin sensitivity . Intrauterine hyperglycaemia $\cdot$ Metabolic syndrome $\cdot$ Prediabetes

\begin{tabular}{|c|c|}
\hline \multicolumn{2}{|c|}{ Abbreviations } \\
\hline $\mathrm{AC}$ & Abdominal circumference \\
\hline BIGTT-AIR & $\begin{array}{l}\text { OGTT-derived index of acute insulin } \\
\text { response }\end{array}$ \\
\hline BIGTT-IS & OGTT-derived index of insulin sensitivity \\
\hline DI & Disposition index \\
\hline DBP & Diastolic BP \\
\hline EPICOM & $\begin{array}{l}\text { Epigenetic Genetic and Environmental } \\
\text { Effects on Growth, Metabolism and } \\
\text { Cognitive Functions in Offspring of } \\
\text { Women with Type } 1 \text { Diabetes study }\end{array}$ \\
\hline GDM & Gestational diabetes mellitus \\
\hline HOMA- $\beta$ & HOMA of insulin secretion \\
\hline IFG & Impaired fasting glucose \\
\hline IGT & Impaired glucose tolerance \\
\hline MetS & Metabolic syndrome \\
\hline NGT & Normal glucose tolerance \\
\hline p-glucose & Plasma glucose \\
\hline SBP & Systolic BP \\
\hline SDS & SD score \\
\hline s-insulin & Serum insulin \\
\hline
\end{tabular}

\section{Introduction}

The metabolic syndrome (MetS) is a cluster of risk factors for cardiovascular disease and type 2 diabetes [1]. It is estimated that around $20-25 \%$ of the world's population meet the criteria for MetS [2]. The prevalence of the MetS increases with age [1], but along with a rising prevalence of overweight among children and adolescents MetS is becoming more prevalent in young age groups [3]. Overweight in childhood and adolescence tends to persist into adulthood and is associated with subsequent adverse health outcomes [4]. Recently, it has been suggested that environmental exposures during fetal life and early infancy may influence metabolic risk in later life [5].

The offspring of mothers with diabetes may display excess fetal growth or macrosomia [6] as initially suggested in the socalled Pedersen hypothesis [7]; maternal hyperglycaemia results in fetal hyperglycaemia, hyperinsulinaemia and thus overgrowth. Other contributing factors to fetal macrosomia are maternal overweight/obesity [8].
The potential clinical implication of intrauterine hyperglycaemia and fetal overgrowth became apparent after studies revealed that exposure to maternal diabetes could have long-term effects on offspring in terms of obesity [9-16], hypertension $[10,17]$ and impaired glucose metabolism [18-21]. However, a number of previous studies examined offspring of women with gestational diabetes mellitus (GDM) [9-11] and type 2 diabetes [14], where maternal obesity is a prominent feature. Studies in childhood of the offspring of women with type 1 diabetes reported an increased risk of overweight/ obesity $[12,13]$ and inflammatory markers [22], whereas studies in adulthood also found defects in glucose metabolism [19, 21, 23]. However, two recent studies of pre-pubertal children of women with type 1 diabetes reported no direct contribution of maternal type 1 diabetes to childhood overweight [24, 25].

Studies of adolescent offspring are scarce and, to our knowledge, associations between maternal $\mathrm{HbA}_{1 \mathrm{c}}$ levels during pregnancy and long-term metabolic outcomes in adolescent offspring of mothers with type 1 diabetes in a prospective setting have not previously been studied.

We aimed to investigate: (1) metabolic risk factors, prevalence of the MetS and pre-diabetes (defined as the presence of impaired fasting glucose [IFG] and/or impaired glucose tolerance [IGT]) in adolescent offspring of mothers with type 1 diabetes in comparison with offspring of non-diabetic mothers; and (2) insulin sensitivity and insulin secretion in these two groups.

\section{Methods}

\section{Study design}

The Epigenetic, Genetic and Environmental Effects on Growth, Metabolism and Cognitive Functions in Offspring of Women with Type 1 Diabetes (EPICOM) study is a prospective nationwide follow-up study in Denmark focusing on the long-term effects of intrauterine diabetic environment. The EPICOM study group consists of adolescent offspring born to mothers with type 1 diabetes between the years 1993 and 1999. During this period, all pregnancies in women with type 1 diabetes in Denmark were prospectively reported to a national register in the Danish Diabetes Association. The register contains detailed information on maternal demography, diabetes status and pregnancy outcome. The women delivered in eight centres: four university hospitals (Copenhagen, Aarhus, Aalborg, Odense) and four county hospitals (Esbjerg, Fredericia, Herning, Hilleroed) with a special interest in diabetes and pregnancy. Data were collected after each delivery by one to three caregivers per centre. The inclusion criterion was delivery after 24 completed weeks of gestation. The women entering the study were all judged as having type 1 diabetes by their caregivers and were on insulin treatment 
before conception. Most of the women were normal weight and the mean diabetes duration was 12 years. The coverage of the cases reported from the centres was $75-93 \%$ evaluated by alternative local data sources [6].

The current study is a follow-up of the offspring from this register (index offspring), compared with a group of offspring of non-diabetic mothers from the background population (control offspring). The study protocol was in accordance with the Declaration of Helsinki and approved by the regional ethics committee (M-20110239). Written informed consent was obtained either from the parents (if the participants were below 18 years of age) or the participants themselves.

\section{Study participants}

The Danish Diabetes Association register consists of 1,215 records of index offspring. For this follow-up study, only singletons and only the first child per mother were included $(n=$ 965). Among the cases fulfilling the inclusion criteria we were not able to identify either the mother or the child in 111 cases. Reasons for this were missing personal identification numbers $(n=30)$ or missing offspring birth date in the Danish Diabetes Association register $(n=10)$, unlisted addresses in The Danish Central Office of Civil Registration or residence outside of Denmark $(n=40)$, or that either the mother or child were not alive at the time of recruitment $(n=31)$. Furthermore, 108 women had chosen a so-called research protected status. Thus, 746 index offspring from the original cohort were eligible for the follow-up examination and invited to participate in the study (Fig. 1). Index offspring were invited with a letter addressed to the mother. Control offspring were identified

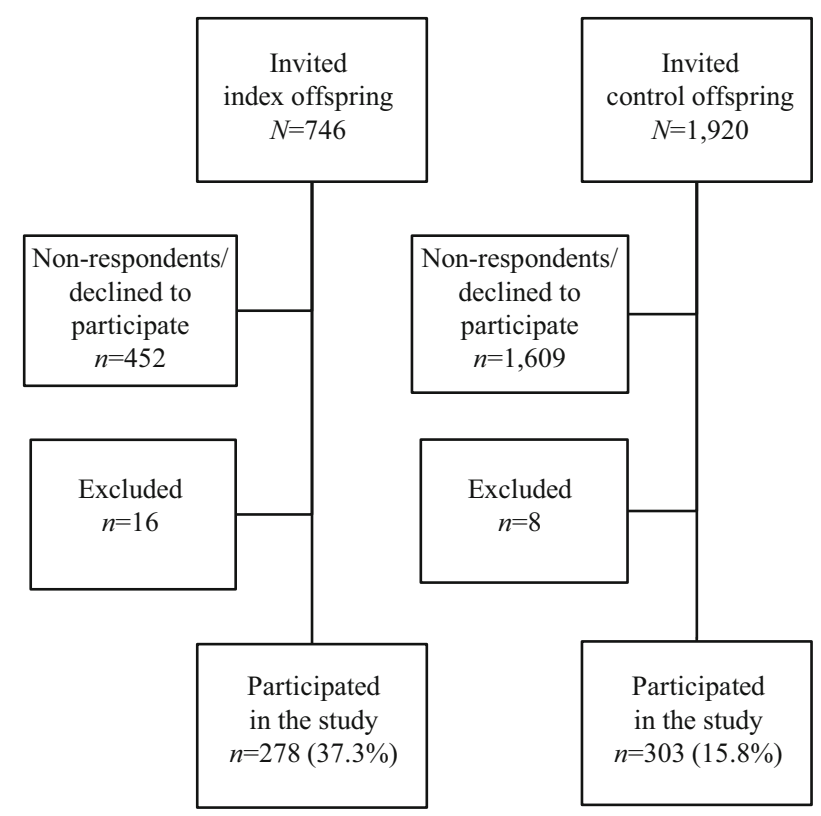

Fig. 1 Flowchart of the study through The Danish Central Civil Registration System and matched with respect to date of birth, sex and place of residence (postal code) as an indirect marker of the socioeconomic status. Between one to five control offspring per all eligible index offspring were invited to participate in the study either with a letter directly (if they were over 18 years of age) or with a letter addressed to the mother. In case of no response two reminders were sent within 6-8 weeks.

\section{Variables}

Main outcomes Height and weight SD scores (SDS) were calculated using a Danish normal reference material [26]. BMI was calculated as weight in kilograms divided by height in metres squared and BMI-SDS was calculated using the Danish normal reference curves [26]. Abdominal circumference (AC) SDS was calculated using British normal reference material [27], which provides reference curves for adolescents up to 17 years of age. AC for offspring aged 17 years and older was used directly. Indices of insulin sensitivity and secretion were derived using data obtained from the OGTT. Insulin sensitivity was evaluated by the OGTT-derived index of insulin sensitivity (BIGTT-IS) [28] and fasting-derived HOMA-IR [29]. To evaluate insulin secretion, we calculated OGTTderived index of acute insulin response (BIGTT-AIR) [28] and the fasting-derived HOMA of insulin secretion (HOMA- $\beta$ ) [29]. Insulin secretion corrected for insulin sensitivity - the disposition index (DI) - was calculated as BIGTTIS multiplied by BIGTT-AIR. Details are given in electronic supplementary material (ESM) Table 1.

We defined prediabetes and diabetes according to the WHO 1999 criteria [30]. Prediabetes was defined as the presence of IFG (fasting plasma [p-]glucose $\geq 6.1$ and $<7.0 \mathrm{mmol} / \mathrm{l}$ with 2 -h p-glucose $<7.8 \mathrm{mmol} / \mathrm{l}$ ) and/or IGT (fasting p-glucose $<7.0 \mathrm{mmol} / \mathrm{l}$ with 2 -h p-glucose $\geq 7.8$ and $<11.1 \mathrm{mmol} / \mathrm{l})$. Diabetes was defined as fasting p-glucose $\geq 7.0 \mathrm{mmol} / 1$ and/or 2 -h p-glucose $\geq 11.1 \mathrm{mmol} / 1$.

MetS in offspring $<16$ years of age was diagnosed according to the International Diabetes Federation 2007 criteria [31], which include abdominal obesity (assessed by sex- and agespecific AC percentiles) plus any two or more of four additional metabolic risk factors: elevated triacylglycerols $\geq 1.7 \mathrm{mmol} / 1$, low HDL-cholesterol $<1.03 \mathrm{mmol} / \mathrm{l}$, high BP $(\geq 130$ systolic and/or $\geq 85$ diastolic) and increased fasting plasma glucose $(\geq 5.6 \mathrm{mmol} / \mathrm{l})$. Offspring who were aged 16 years and older were diagnosed with the adult MetS criteria [2].

Exposure variables The main exposure variable was exposure to the intrauterine diabetic environment (defined as the offspring of women with type 1 diabetes - the index offspring).

Potential confounders and effect modifiers Offspring age and sex were included in the analyses unless the studied 
outcomes were presented as age- and sex-specific SDS. Pubertal development was assessed by the Tanner method based on breast development in girls [32] and genital development and measurement of testicular volume in boys [33]. Maternal pre-pregnancy BMI was taken either from the Danish Diabetes Association register (index offspring) or was found retrospectively from the obstetric medical records (control offspring). We used peri-conceptional $\mathrm{HbA}_{1 \mathrm{c}}$ (first trimester $\mathrm{HbA}_{1 \mathrm{c}}$ and in case of missing value the latest prepregnancy $\mathrm{HbA}_{1 \mathrm{c}}$ ), second trimester $\mathrm{HbA}_{1 \mathrm{c}}$ and the third trimester $\mathrm{HbA}_{1 \mathrm{c}}$ to investigate the effect of maternal glycaemic control on long-term metabolic outcomes in the offspring. All participants and their parents were asked to fill in a questionnaire addressing social aspects and education levels. Educational level of the mother was derived as the sum of years in school plus years of higher education.

The laboratory staff member responsible for the analyses of blood samples was blinded to the status of diabetes exposure in the offspring. The caregivers performing the clinical examinations were responsible for the recruitment of the participants and were, therefore, not blinded.

\section{Clinical examination}

The clinical examinations took place in three different university hospitals in Denmark (Copenhagen, Odense, and Aarhus) from April 2012 until October 2013. All procedures were identical for index offspring and control offspring and across the three centres. Participants were studied after an overnight fast and the examinations detailed below were performed.

Anthropometric measurements All measurements except height were performed three times and the mean value was used for the analyses. Height was measured to the nearest $0.1 \mathrm{~cm}$ without shoes with a permanently affixed stadiometer in centimetres. Weight was measured to the nearest $0.1 \mathrm{~kg}$ on a calibrated personal weight in kilograms. Measurement of AC was done using a tape measure to the nearest $0.5 \mathrm{~cm}$ midway between the arcus costae and crista iliaca after exhalation. Blood pressure was measured in the supine position after $5 \mathrm{~min}$ of rest using a digital BP metre (Omron 705 IT; Omron Healthcare, Hoofddorp, the Netherlands).

Biochemical testing and standard 2 h OGTT OGTT was performed with a glucose load of $1.75 \mathrm{~g} / \mathrm{kg}$ body weight up to a total of $75 \mathrm{~g}$. Venous plasma was drawn from an antecubital vein at 0,30 and $120 \mathrm{~min}$ after glucose administration to determine plasma glucose and serum insulin levels. Furthermore, at $0 \mathrm{~min}$, venous blood was drawn to measure plasma lipids and $\mathrm{HbA}_{1 \mathrm{c}}$. OGTT was not performed if the child had already been diagnosed with diabetes.

\section{Biochemical analyses}

Glucose was measured in venous plasma with a hexokinaseglucose-6-phosphate dehydrogenase assay (Abbott Diagnostics, Abbott Park, IL, USA). Serum insulin was measured by ELISA using dual-monoclonal antibodies (ALPCO Diagnostics, Salem, NH, USA). Lipids were measured by enzymatic calorimetric analysis, end-up reaction (Abbott) and $\mathrm{HbA}_{1 \mathrm{c}}$ was measured by cation-exchange HPLC (G8 analyzer; Tosoh Bioscience, San Francisco, CA, USA). Analyses of maternal $\mathrm{HbA}_{1 \mathrm{c}}$ between 1993 and 1999 were measured on local assays. Correction was made to a common standard by multiplying the $\mathrm{HbA}_{1 \mathrm{c}}$ value with a correction factor (mean of the reference values for a standard assay divided by the mean of the reference values for the given assay). The assays were subjected to thorough centralised national quality control during this period.

\section{Statistical analyses}

Continuous variables with symmetric distribution are presented as means and SDs; continuous variables with skewed distribution are presented as medians and interquartile ranges. We fitted a linear model for each of the outcomes with index/control status as an independent variable reporting the differences between the groups with $95 \%$ CIs and $p$ values. The assumption of normality of the residuals was checked. Data with skewed distribution were $\log _{\mathrm{e}}$-transformed and relative differences between the groups are given as a percentage difference. Analyses were adjusted for sex, age (except for the SDS-corrected indices), Tanner stage and maternal prepregnancy BMI. Both systolic BP (SBP) and diastolic BP (DBP) were additionally adjusted for height, which is a strong predictor for BP in childhood and adolescence [34].

BIGTT-AIR and BIGTT-IS were not adjusted for sex, because sex (along with BMI) is incorporated in the calculations of these indices. Calculation of HOMA-IR and HOMA- $\beta$ does not include BMI and these two variables were, therefore, additionally adjusted for offspring BMI. We analysed the effect of maternal $\mathrm{HbA}_{1 \mathrm{c}}$ levels on metabolic outcomes in the offspring by multiple regression using a linear model with $\mathrm{HbA}_{1 \mathrm{c}}$ as an independent continuous variable. Analyses were done for peri-conceptional second and third trimester $\mathrm{HbA}_{1 \mathrm{c}}$. Results are reported as change per percentage of $\mathrm{HbA}_{1 \mathrm{c}}$.

Comparison of categorical variables was done using Fisher's exact test. All statistical analyses were performed using the statistical program $\mathrm{R}$, version 3.0.3 [35].

\section{Results}

Maternal/fetal baseline characteristics and offspring anthropometrics and metabolic characteristics at follow-up are shown 
in Tables 1 and 2, and in Fig. 2. Additional information is available in the ESM.

A total of 278 index offspring (37.3\%) with mean age 16.7 years (range 13.0-19.8 years) agreed to participate in the study, while $452(60.6 \%)$ either did not respond or did not wish to participate. Sixteen index offspring $(2.1 \%)$ were excluded due to the following reasons: maternal diagnosis of type 1 diabetes was later reclassified to either MODY or type 2 diabetes $(n=12)$, the mother had no contact with the child $(n=2)$, drug abuse $(n=1)$ or pregnancy at the time of recruitment $(n=1)$. Baseline data on index participants and nonparticipants are given in ESM Table 2. Of 1,920 invited matched control offspring, 303 (15.8\%) with mean age 16.8 years (range 13.5-20.4 years) participated in the study, while $83.8 \%$ either did not respond or did not wish to participate. We excluded eight control offspring due to the following reasons: they were adopted and had no contact with their biological mothers $(n=4)$, their place of birth was outside of Denmark $(n=1)$ or obstetric medical records revealed that their mothers had gestational diabetes $(n=3)$.

All participants were born in Denmark and most of them were of white European ethnicity. Only 1.2\% (three index and four control offspring) belonged to other ethnic groups. Baseline characteristics for both index and control offspring are shown in Table 1. Index offspring had on average 1.8 higher birthweight SDS (corrected for sex and gestational age) than control offspring but there were no significant differences as regards maternal age and BMI.

Metabolic and anthropometric characteristics of the participants by index/control status at follow-up are shown in Table 2. Data on anthropometrics are $100 \%$ complete. The OGTT data are $>95 \%$ complete.
Index offspring had on average 0.44 higher BMI-SDS after adjusting for pubertal development and maternal prepregnancy BMI compared with controls. Both SBP and DBP were increased in index offspring $(1.9 \mathrm{mmHg}$ and $1.3 \mathrm{mmHg}$, respectively) compared with controls. Furthermore, index offspring had lower HDL-cholesterol levels than controls in crude analyses, but the differences disappeared in adjusted analyses.

During the OGTT, index offspring had higher levels of p-glucose and s-insulin in both fasting and postload stage. Only levels of fasting p-glucose remained significantly increased in adjusted analyses. Levels of s-insulin remained significantly increased during the whole OGTT, even after adjusting for confounders, and were $15 \%$ higher than those in control offspring.

BIGTT-S was decreased (1.6 units) and HOMA-IR was increased (by 10\%) in index offspring, as was BIGTT-AIR. No significant differences in HOMA- $\beta$ were observed between the two groups. The DI was lower in index offspring compared with controls.

All components of the MetS were more prevalent in index offspring than in control offspring (ESM Table 3). Overall, the MetS tended to be more frequent in index offspring than in controls $(2.8 \%$ vs $0.7 \% ; p=0.054)$. The distribution of glucose tolerance groups was less favourable among index offspring, with $15.4 \%$ prediabetes vs $8.1 \%$ in controls ( $p=0.011$; ESM Table 4). Two index offspring were previously diagnosed with type 1 diabetes. One index offspring was diagnosed with diabetes during OGTT (fasting p-glucose 8.3 and 2-h p-glucose $13.9 \mathrm{mmol} / \mathrm{l})$. There was no significant association of BMISDS with prediabetes; offspring with prediabetes had on average 0.06 higher BMI-SDS than offspring without

Table 1 Baseline characteristics of mothers and their offspring by index/control status

\begin{tabular}{|c|c|c|c|c|c|}
\hline \multirow[t]{2}{*}{ Characteristic } & \multirow{2}{*}{$\begin{array}{l}\text { Index mothers and } \\
\text { their offspring } \\
n=278 \\
\text { Mean } \pm \text { SD }\end{array}$} & \multirow{2}{*}{$\begin{array}{l}\text { Control mothers } \\
\text { and their offspring } \\
n=303 \\
\text { Mean } \pm \text { SD }\end{array}$} & \multicolumn{3}{|c|}{$\begin{array}{l}\text { Differences between mothers and their offspring by } \\
\text { index/control status }\end{array}$} \\
\hline & & & Difference & $95 \% \mathrm{CI}$ & $p$ value \\
\hline Parity & 1.54 & 1.75 & & & \\
\hline Maternal pre-pregnancy age (years) & $29.2 \pm 4.3$ & $29.2 \pm 4.1$ & 0.0 & $(-0.7$ to 0.7$)$ & 0.946 \\
\hline Maternal pre-pregnancy BMI $\left(\mathrm{kg} / \mathrm{m}^{2}\right)$ & $23.5 \pm 3.2$ & $23.3 \pm 4.0$ & 0.2 & $(-0.5$ to 0.9$)$ & 0.577 \\
\hline Duration of diabetes (years) & $12.4 \pm 8.3$ & & & & \\
\hline Peri-conceptional $\mathrm{HbA}_{1 \mathrm{c}}(\%)$ & $7.3 \pm 1.1$ & & & & \\
\hline $\mathrm{HbA}_{1 \mathrm{c}}$ in second trimester $(\%)$ & $6.6 \pm 1.0$ & & & & \\
\hline $\mathrm{HbA}_{1 \mathrm{c}}$ in third trimester $\mathrm{HbA}_{1 \mathrm{c}}(\%)$ & $6.7 \pm 1.0$ & & & & \\
\hline Peri-conceptional $\mathrm{HbA}_{1 \mathrm{c}}(\mathrm{mmol} / \mathrm{mol})$ & $56.0 \pm 12.2$ & & & & \\
\hline $\mathrm{HbA}_{1 \mathrm{c}}$ in second trimester $(\mathrm{mmol} / \mathrm{mol})$ & $48.7 \pm 10.7$ & & & & \\
\hline $\mathrm{HbA}_{1 \mathrm{c}}$ in third trimester $(\mathrm{mmol} / \mathrm{mol})$ & $49.8 \pm 10.8$ & & & & \\
\hline Birthweight SDS & $1.83 \pm 2.05$ & $0.01 \pm 0.95$ & 1.82 & (1.53 to 2.12$)$ & $<0.001$ \\
\hline
\end{tabular}

Data are presented as means $\pm \mathrm{SD}$

Differences between the groups are reported as estimates from linear regression with $95 \% \mathrm{CI}$ and $p$ values 


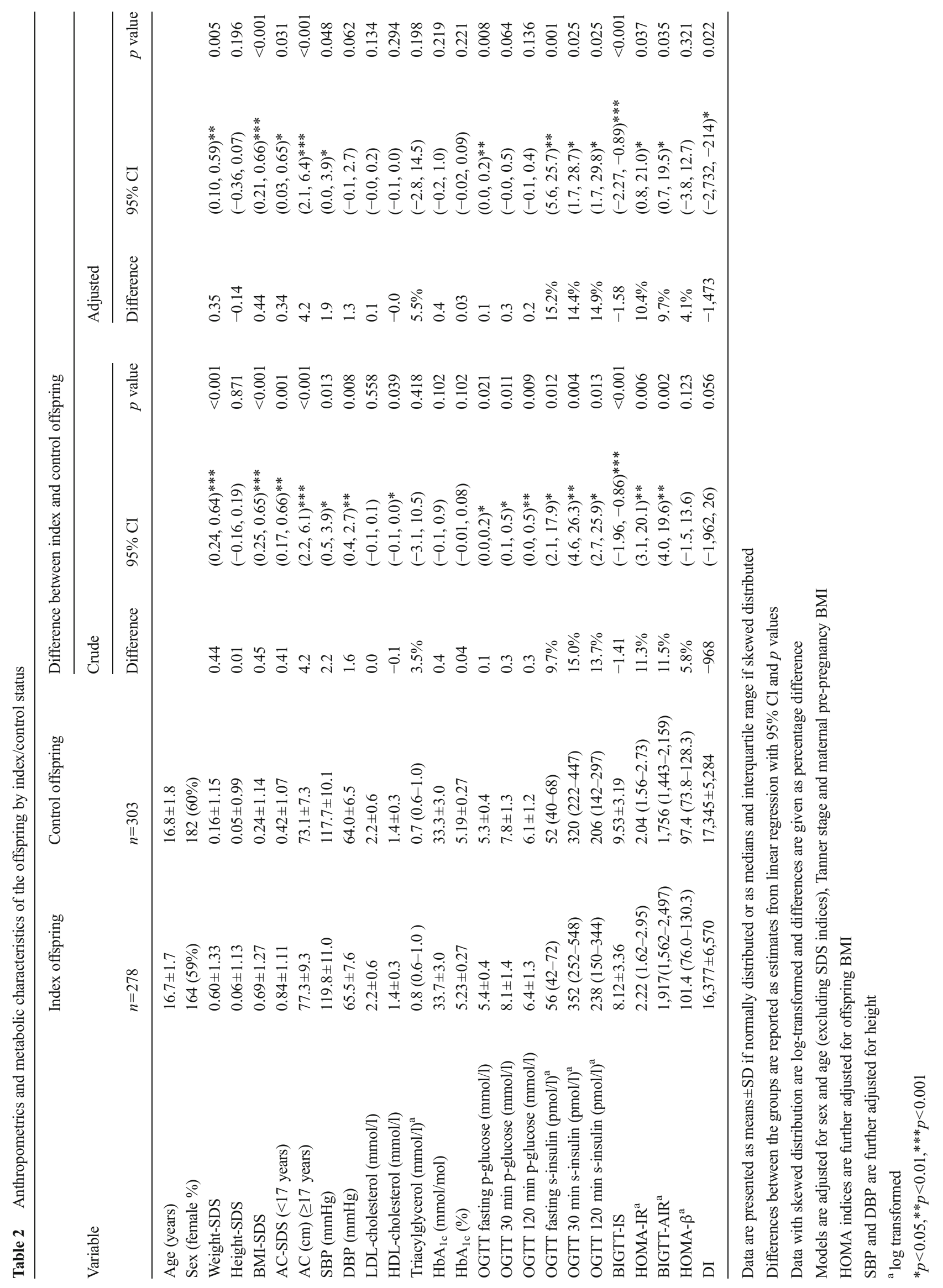


Fig. 2 Percentage of index offspring (black bars) and control offspring (white bars) by the presence of (a) components of MetS and (b) glucose tolerance status $\mathbf{a}$

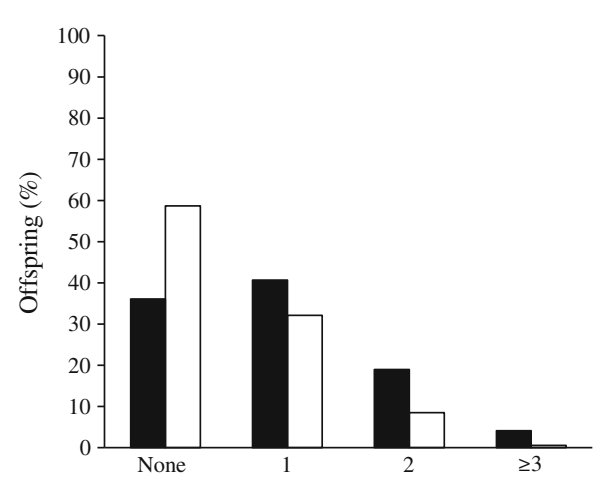

b

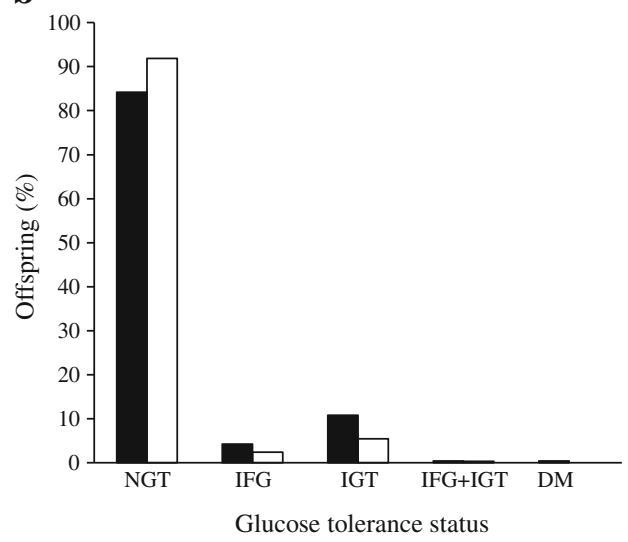

$(p=0.730)$. The lack of association of BMI-SDS with prediabetes was similar for index and control offspring.

In univariate analyses, BMI-SDS increased with periconceptional $\mathrm{HbA}_{1 \mathrm{c}}$ levels: BMI-SDS 0.14 (95\% CI -0.01, 0.28) per $\% \mathrm{HbA}_{1 \mathrm{c}}$. The effect of peri-conceptional $\mathrm{HbA}_{1 \mathrm{c}}$ weakened when adjusting for stage of pubertal development and maternal pre-pregnancy BMI: BMI-SDS 0.06 (95\% CI $-0.09,0.21)$ per $\% \mathrm{HbA}_{1 \mathrm{c}}$. Analyses using third trimester $\mathrm{HbA}_{1 \mathrm{c}}$ showed increased BMI-SDS with increasing $\mathrm{HbA}_{1 \mathrm{c}}$ levels: $0.18(95 \%$ CI $0.02,0.34)$ per $\% \mathrm{HbA}_{1 \mathrm{c}}$, but this was no longer statistically significant when adjusting for stage of pubertal development and maternal pre-pregnancy BMI: 0.13 $(95 \% \mathrm{CI}-0.06,0.31)$ per $\% \mathrm{HbA}_{1 \mathrm{c}}$. Likewise, second trimester $\mathrm{HbA}_{1 \mathrm{c}}$ did not significantly affect the results (data not shown). There were no significant associations between $\mathrm{HbA}_{1 \mathrm{c}}$ levels in pregnancy and offspring BP, lipids, insulin sensitivity and insulin secretion (ESM Tables 5 and 6). Adjusting the data for maternal education did not significantly change the results.

\section{Discussion}

This well-characterised, large and prospectively identified cohort of offspring of mothers with type 1 diabetes had a higher frequency of metabolic risk factors included in the MetS than offspring of the same age born to non-diabetic mothers. Furthermore, index offspring had decreased insulin sensitivity and insufficient compensatory insulin secretion compared with offspring in the control group, resulting in a higher prevalence of prediabetes. Significant associations between these outcomes and maternal $\mathrm{HbA}_{1 \mathrm{c}}$ levels in pregnancy could not be demonstrated.

Several studies have previously reported an association between maternal diabetes and increased risk of overweight and other metabolic disorders in the offspring. Studies of Pima Indians showed that both diabetes and elevated 2-h blood glucose during OGTT in pregnancy were strong predictors of overweight and type 2 diabetes in the offspring [14].
Pima Indians have specific genetic traits with a high prevalence of overweight and type 2 diabetes, which might have influenced the results. However, within the same family, offspring born after the mother was diagnosed with diabetes had a much greater risk of being obese and of developing type 2 diabetes at an early age than offspring born before the mother was diagnosed with diabetes [36]. This finding could indicate that exposure to the intrauterine diabetic environment is an important determinant of obesity and type 2 diabetes in addition to the genetic predisposition. It has been suggested that associations between maternal diabetes and effects on the offspring may be related to intrauterine hyperglycaemia per se and not to the type of maternal diabetes. A similar prevalence of IGT in offspring aged 1-9 years of mothers with both type 1 diabetes and GDM has been reported [18]. In a Danish study of young adults, the prevalence of prediabetes/type 2 diabetes was $>10 \%$ in the offspring of mothers with type 1 diabetes and $>20 \%$ in offspring born to mothers with GDM [19].

In cohorts consisting exclusively of offspring of women with type 1 diabetes, Rijpert et al [25] found a similar prevalence of overweight in offspring aged 6-8 years $(n=213)$ of women with type 1 diabetes with adequate glycaemic control during the pregnancy and a reference population. Lindsay et al [12] reported increased BMI, AC and adiposity at 7 years $(n=100)$, but no differences in plasma glucose or insulin levels. In a large study by Hummel et al [24] of 578 offspring of mothers and 636 offspring of fathers with type 1 diabetes, aged up to 8 years of age, no evidence was found for maternal type 1 diabetes to be an independent predictor for overweight in childhood. In the study by Weiss et al [13], offspring aged $5-15$ years $(n=75)$ of mothers with type 1 diabetes had significantly higher BMI and incidence of risk factors predictive for type 2 diabetes, including increased postload glucose, fasting and postload insulin, and insulin resistance, than children born to metabolically healthy mothers. These findings are in accordance with our results. A summarised overview of the previously published studies in offspring of women with type 1 diabetes is given in the ESM Table 7. It could be 
speculated that impairment in insulin sensitivity and insulin secretion in offspring exposed to diabetic intrauterine environment appear later in life. Silverman et al [20] reported an increase in the prevalence of IGT in a mixed cohort of offspring exposed to the intrauterine diabetic environment first after 10 years of age.

The mechanism behind glycaemic disorders in offspring exposed to the intrauterine diabetic environment are multifactorial and still only sparsely understood, but impairment of both insulin secretion and insulin sensitivity seem to be two important pathophysiological mechanisms [23,37]. Low DI is associated with IGT and is predictive of type 2 diabetes development [38] as a result of exhaustion of beta cells and failure to maintain increased insulin secretion. Bush et al [37] found an inverse association between maternal glucose concentration in pregnancy and insulin sensitivity, and a positive association between maternal glucose concentration in pregnancy and beta cell responsitivity in 5-10 years old offspring of mothers with GDM. We observed lower insulin sensitivity and increased insulin secretion along with lower DI in index offspring compared with controls. This is in accordance with the findings of Kelstrup et al [23], though an increase in insulin secretion was not reported in that study, maybe because beta cell exhaustion had taken place in adulthood. How such impairment of insulin sensitivity and insulin secretion comes into action is still unresolved, but fundamental epigenetic changes of the genome may be involved [39]. Based on the developmental origins of disease hypothesis [5], intrauterine development may present a vulnerable time period when the maternal environment can affect the longterm metabolic health of offspring. Our study group has recently reported an association between overall morbidity in index offspring and maternal pre-pregnancy and first trimester $\mathrm{HbA}_{1 \mathrm{c}}$ [40]. However, in the current study we did not find an independent association between $\mathrm{HbA}_{1 \mathrm{c}}$ levels in pregnancy and long-term metabolic outcomes in the offspring.

A major strength of the present study is the large number of offspring of well-characterised mothers with type 1 diabetes, with detailed clinical information available from the pregnancies, including $\mathrm{HbA}_{1 \mathrm{c}}$ levels. Moreover, the longitudinal and prospective study design with a wide range of anthropometric and metabolic outcomes, as well as the large number of matched population controls, make this study unique.

However, some limitations deserve comment. The index offspring had higher participation rate (37\%) than control offspring $(16 \%)$ and if selection is preferential towards healthier controls this may contribute to the type of the results we observed. We did not have information about education and social class for the non-participants, which could be used to evaluate this variable. Furthermore, index mothers might be preferentially interested in health examination of children with potential health problems, which would also bias the study. There is no Danish study addressing the prevalence of prediabetes in children or adolescents, so we do not know whether the prevalence of prediabetes of $8 \%$ is the same for the general adolescent population. The participating index children had on average 0.3 higher birthweight SDS than that of non-participants, potentially favouring the former group with a higher metabolic risk. On the other hand, mothers of non-participant index offspring had slightly higher periconceptional $\mathrm{HbA}_{1 \mathrm{c}}$, which might bias the results towards the null hypothesis. There were no differences in other clinical variables, such as maternal age, BMI, duration of diabetes and gestational age of the offspring (ESM Table 2).

Beside HOMA indexes we used BIGTT indices to evaluate insulin sensitivity and secretion in the offspring. We chose these indices because they incorporate BMI and sex in the calculations, but these indices have not been validated in an adolescent population. The Danish Diabetes Association register does not contain information on maternal pregnancy weight gain and breastfeeding, so we were unable to adjust the linear multivariate analyses for these factors, which could potentially influence the results $[11,24]$. Examination of metabolic health in adolescence is challenging since insulin sensitivity decreases during puberty resulting in a compensatory increase in basal and stimulated insulin secretion [41]. Thus, all analyses were adjusted for Tanner stage along with sex and age in order to eliminate influence of different stages of pubertal development on our analyses. Furthermore, all anthropometric measurements are presented as SDS according to the latest Danish reference curves, which makes the calculations more accurate than in most other studies. However, Danish reference curves for $\mathrm{AC}$ were available only for children $<14$ years of age, so the British AC reference curves providing reference data up to 17 years of age were used.

\section{Conclusion}

Adolescent offspring of mothers with type 1 diabetes had a less favourable metabolic profile and a higher frequency of prediabetes than the background population. Significant associations between these outcomes and maternal $\mathrm{HbA}_{1 \mathrm{c}}$ levels in pregnancy could not be demonstrated.

Acknowledgements Data collection in the original registry was performed by L. Mølsted-Pedersen, J. Klebe, J. Westergaard, M. Møller, N. Hahnemann, H. Gjessing, J. K. Mostrup, K. H. Frandsen, E. Stage, A. Thomsen, T. Lousen, K. R. Petersen, B. Øvlisen, J. Kvetny, H. Poulsen (The Danish Diabetes Working Group for Type 1 Diabetes Pregnancy).

Apart from H. Beck-Nielsen and P. Damm, the original registry working group included A. Frøland, L. Mølsted-Pedersen, J. Klebe and C. E. Mogensen. The Danish Diabetes Association is acknowledged for originally assisting in the creation and funding of the original Danish Diabetes Birth Registry.

Funding This study was supported by a grant from the Danish Council for Independent Research, European Foundation for the Study of 
Diabetes, Lundbeck Foundation, Danielsens Foundation and Beckett Foundation.

Duality of interest RBJ, HBN, PO, CHG, ERM and PD have given talks for Novo Nordisk (NN). PD, PO and ERM are participating in a multinational study in collaboration with $\mathrm{NN}$, and $\mathrm{HBN}$ receives research support from NN. All other authors declare that there is no duality of interest associated with their contribution to this manuscript.

Contribution statement $\mathrm{HBN}$ and PD contributed to the establishment of the original birth registry. $\mathrm{PD}, \mathrm{PO}$ and $\mathrm{DMJ}$ contributed with data collection of this registry. All authors contributed substantially to the planning and design of the current study. BB, SK and ZV performed the clinical examinations of the offspring and data collection. ZV analysed the data and wrote the manuscript. DMJ, PD, CHG, TDC, RBJ, ERM, $\mathrm{BB}$ and SK contributed to the discussion, and editing and revising the manuscript. HBN, $\mathrm{KH}$ and $\mathrm{PO}$ reviewed the manuscript. DMJ is the guarantor of the study. All authors approved the final version.

\section{References}

1. Samson SL, Garber AJ (2014) Metabolic syndrome. Endocrinol Metab Clin N Am 43:1-23

2. International Diabetes Fedetation (2006) The IDF consensus worldwide definition of the metabolic syndrome. IDF, Brussels. Available from www.idf.org/webdata/docs/IDF_Meta_def final. pdf. Accessed 22 May 2014

3. Duncan G, Li S, Zhou X (2004) Prevalence and trends of a metabolic syndrome phenotype among US adolescents, 1999-2000. Diabetes Care 27:2438-2443

4. DiPietro L, Mossberg HO, Stunkard AJ (1994) A 40-year history of overweight children in Stockholm: life-time overweight, morbidity, and mortality. Int J Obes Relat Metab Disord 18:585-590

5. Hochberg Z, Feil R, Constancia M et al (2011) Child health, developmental plasticity, and epigenetic programming. Endocr Rev 32: 159-224

6. Jensen DM, Damm P, Moelsted-Pedersen L et al (2004) Outcomes in type 1 diabetic pregnancies: a nationwide, population-based study. Diabetes Care 27:2819-2823

7. Pedersen J (1961) Pathogenesis of the characteristic features of newborn infants of diabetic women. In: The pregnant diabetic and her newborn. Baltimore, MD, Williams and Wilkins, p 128-137

8. Ehrenberg HM, Mercer BM, Catalano PM (2004) The influence of obesity and diabetes on the prevalence of macrosomia. Am J Obstet Gynecol 191:964-968

9. Hillier T, Pedula K, Schmidt M (2007) Childhood obesity and metabolic imprinting the ongoing effects of maternal hyperglycemia. Diabetes Care 30:2287-2292

10. Wright $C$ (2009) Intrauterine exposure to gestational diabetes, child adiposity, and blood pressure. Am J Hypertens 22:215-220

11. Page KA, Romero A, Buchanan TA, Xiang AH (2014) Gestational diabetes mellitus, maternal obesity, and adiposity in offspring. J Pediatr 164:807-810

12. Lindsay RS, Nelson SM, Walker JD et al (2010) Programming of adiposity in offspring of mothers with type 1 diabetes at age 7 years. Diabetes Care 33:1080-1085

13. Weiss PA, Scholz HS, Haas J et al (2000) Long-term follow-up of infants of mothers with type 1 diabetes. Diabetes Care 23:905-911

14. Dabelea D, Knowler WC, Pettitt DJ (2000) Effect of diabetes in pregnancy on offspring: follow-up research in the Pima Indians. J Matern Fetal Med 9:83-88

15. Clausen TD, Mathiesen ER, Hansen T et al (2009) Overweight and the metabolic syndrome in adult offspring of women with diet-treated gestational diabetes mellitus or type 1 diabetes. $\mathrm{J}$ Clin Endocrinol Metab 94:2464-2470

16. Philipps LH, Santhakumaran S, Gale C et al (2011) The diabetic pregnancy and offspring BMI in childhood: a systematic review and meta-analysis. Diabetologia 54:1957-1966

17. Aceti A, Santhakumaran S, Logan KM et al (2012) The diabetic pregnancy and offspring blood pressure in childhood: a systematic review and meta-analysis. Diabetologia 55:3114-3127

18. Plagemann A, Harder T, Kohlhoff R et al (1997) Glucose tolerance and insulin secretion in children of mothers with pregestational IDDM or gestational diabetes. Diabetologia 40:1094-1100

19. Clausen TD, Mathiesen ER, Hansen T et al (2008) High prevalence of type 2 diabetes and pre-diabetes in adult offspring of women with gestational diabetes mellitus or type 1 diabetes: the role of intrauterine hyperglycemia. Diabetes Care 31:340-346

20. Silverman BL, Metzger BE, Cho NH, Loeb CA (1995) Impaired glucose tolerance in adolescent offspring of diabetic mothers. Relationship to fetal hyperinsulinism. Diabetes Care 18:611-617

21. Sobngwi E, Boudou P, Mauvais-Jarvis F et al (2003) Effect of a diabetic environment in utero on predisposition to type 2 diabetes. Lancet 361:1861-1865

22. Manderson JG, Mullan B, Patterson CC et al (2002) Cardiovascular and metabolic abnormalities in the offspring of diabetic pregnancy. Diabetologia 45:991-996

23. Kelstrup L, Damm P, Mathiesen ER et al (2014) Insulin resistance and impaired pancreatic $\beta$-cell function in adult offspring of women with diabetes in pregnancy. J Clin Endocrinol Metab 98:3793-3801

24. Hummel S, Pflüger M, Kreichauf S (2009) Predictors of overweight during childhood in offspring of parents with type 1 diabetes. Diabetes Care 32:921-925

25. Rijpert M, Evers IM, de Vroede MAMJ et al (2009) Risk factors for childhood overweight in offspring of type 1 diabetic women with adequate glycemic control during pregnancy: nationwide follow-up study in the Netherlands. Diabetes Care 32:2099-2104

26. Tinggaard J, Aksglaede L, Sørensen K et al (2014) The 2014 Danish references from birth to 20 years for height, weight and body mass index. Acta Paediatr 103:214-224

27. Pan H, Cole T (2012) LMSgrowth - growth references based on the LMS method. Version 2.77. Microsoft Excel add-in to access growth references. Available from www.healthforallchildren. com/?product=lmsgrowth. Accessed 6 Nov 2014

28. Hansen T, Drivsholm T, Urhammer SA et al (2007) The BIGTT test: a novel test for simultaneous measurement of pancreatic beta-cell function, insulin sensitivity, and glucose tolerance. Diabetes Care 30:257-262

29. Matthews DR, Hosker JP, Rudenski AS et al (1985) Homeostasis model assessment: insulin resistance and beta-cell function from fasting plasma glucose and insulin concentrations in man. Diabetologia 28:412-419

30. WHO/IDF (2006) Report of a WHO/IDF consultation. Definition and diagnosis of diabetes mellitus and intermediate hyperglycaemia. WHO, Geneva. Available from www.who.int/ diabetes/publications/diagnosis_diabetes2006/en/. Accessed 24 May 2014

31. Zimmet P, Alberti G, Kaufman F, Tajima N (2007) The metabolic syndrome in children and adolescents. Lancet 2059-2061

32. Marshall WA, Tanner JM (1969) Variations in pattern of pubertal changes in girls. Arch Dis Child 44:291-303

33. Marshall WA, Tanner JM (1970) Variations in the pattern of pubertal changes in boys. Arch Dis Child 45:13-23

34. Rosner B, Cook N, Portman R et al (2008) Determination of blood pressure percentiles in normal-weight children: some methodological issues. Am J Epidemiol 167:653-666

35. R Core Team (2014) R: a language and environment for statistical computing, version 3.0.3. R Foundation for Statistical Computing, Vienna, Austria. www.R-project.org 
36. Dabelea D, Hanson RL, Lindsay RS et al (2000) Intrauterine exposure to diabetes conveys risks for type 2 diabetes and obesity: a study of discordant sibships. Diabetes 49:22082211

37. Bush NC, Chandler-Laney PC, Rouse DJ et al (2011) Higher maternal gestational glucose concentration is associated with lower offspring insulin sensitivity and altered beta-cell function. J Clin Endocrinol Metab 96:E803E809

38. Utzschneider K, Prigeon R (2009) Oral disposition index predicts the development of future diabetes above and beyond fasting and 2-h glucose levels. Diabetes Care 32: 335-341

39. Portha B, Fournier A, Kioon MDA et al (2014) Early environmental factors, alteration of epigenetic marks and metabolic disease susceptibility. Biochimie 97:1-15

40. Knorr S, Stochholm K, Vlachová Z et al (2015) Multisystem morbidity and mortality in offspring of women with type 1 diabetes (The EPICOM Study): a register-based prospective cohort study. Diabetes Care dc142907

41. Goran MI, Gower BA (2001) Longitudinal study on pubertal insulin resistance. Diabetes 50:2444-2450 